\title{
Article \\ Some Algebraic Polynomials and Topological Indices of Octagonal Network
}

\author{
Young Chel Kwun ${ }^{1}$, Waqas Nazeer ${ }^{2}$, Mobeen Munir ${ }^{2}$ and Shin Min Kang 3,4,* \\ 1 Department of Mathematics, Dong-A University, Busan 604-714, Korea; yckwun@dau.ac.kr \\ 2 Division of Science and Technology, University of Education, Lahore 54000, Pakistan; \\ nazeer.waqas@ue.edu.pk (W.N.); mmunir@ue.edu.pk (M.M.) \\ 3 Department of Mathematics and RINS, Gyeongsang National University, Jinju 52828, Korea \\ 4 Center for General Education, China Medical University, Taichung 40402, Taiwan \\ * Corresponding author: smkang@gnu.ac.kr
}

\begin{abstract}
M-polynomial of different molecular structures helps to calculate many topological indices. A topological index of graph $G$ is a numerical parameter related to $G$ which characterizes its molecular topology and is usually graph invariant. In the field of quantitative structure-activity (QSAR) quantitative structure-activity structure-property (QSPR) research, theoretical properties of the chemical compounds and their molecular topological indices such as the Zagreb indices, Randic index, Symmetric division index, Harmonic index, Inverse sum index, Augmented Zagreb index, multiple Zagreb indices etc. are correlated. In this report, we compute closed forms of M-polynomial, first Zagreb polynomial and second Zagreb polynomial of Octagonal network. From the M-polynomial we recover some degree-based topological indices for Octagonal network. Moreover, we give a graphical representation of our results.
\end{abstract}

Keywords: M-polynomial; Zagreb polynomial; topological index; network

\section{Introduction}

Chemical reaction network theory is an area of applied mathematics that attempts to model the behavior of real-world chemical systems. Since its foundation in the 1960s, it has attracted a growing research community, mainly due to its applications in biochemistry and theoretical chemistry. It has also attracted interest from pure mathematicians due to the problems that arise from the mathematical structures.

Cheminformatics is an emerging field in which quantitative structure-activity (QSAR) and Structure-property (QSPR) relationships predict the biological activities and properties of nanomaterial see [1-4]. In these studies, some physcio-chemical properties and topological indices are used to predict bioactivity of the chemical compounds see [5-7].

The branch of chemistry which deals with the chemical structures with the help of mathematical tools is called mathematical chemistry. Chemical graph theory is that branch of mathematical chemistry which applies graph theory to mathematical modeling of chemical phenomena. In chemical graph theory a molecular graph is a simple graph (having no loops and multiple edges) in which atoms and chemical bonds between them are represented by vertices and edges respectively. A graph $G(V, E)$ with vertex set $\mathrm{V}(\mathrm{G})$ and edge set $\mathrm{E}(\mathrm{G})$ is connected, if there exist a connection between any pair of vertices in $G$. 
A network is simply a connected graph having no multiple edges and loops. A chemical graph is a graph whose vertices denote atoms and edges denote bonds between those atoms of any underlying chemical structure. The degree of a vertex is the number of vertices which are connected to that fixed vertex by the edges. In a chemical graph the degree of any vertex is at most 4 . The distance between two vertices $\mathrm{u}$ and $\mathrm{v}$ is denoted as $d(u, v)=d_{G}(u, v)$ and is the length of shortest path between $u$ and $v$ in graph $G$. The number of vertices of $G$, adjacent to a given vertex $v$, is the "degree" of this vertex, and will be denoted by $d_{v}$. The concept of degree in graph theory is closely related (but not identical) to the concept of valence in chemistry. For details on basics of graph theory, any standard text such as [3] can be of great help.

Several algebraic polynomials have useful applications in chemistry such as Hosoya Polynomial (also called Wiener polynomial) [8] that plays a vital role in determining distance-based topological indices. Among other algebraic polynomials, M-polynomial [4], introduced in 2015 plays the same role in determining many degree-based topological indices.

Definition 1. Let $\mathrm{G}$ be a simple connected graph. The M-polynomial of $\mathrm{G}$ is defined as:

$$
M(G, x, y)=\sum_{\delta \leq i \leq j \leq \Delta} m_{i j}(G) x^{i} y^{j}
$$

Where $\delta=\operatorname{Min}\left\{d_{v} \mid v \in \mathrm{V}(\mathrm{G})\right\}, \Delta=\operatorname{Max}\left\{d_{v} \mid v \in \mathrm{V}(\mathrm{G})\right\}$, and $m_{i j}(G)$ is the edge $v u \in E(G)$

such that $\left\{d_{v}, d_{u}\right\}=\{i, j\}$.

This polynomial is one of the key areas of interest in computational aspects of materials. From this M-polynomial, we can calculate many topological indices. M-polynomial of different molecular structures has been computed in [9-12]. The topological index of a molecule structure can be considered as a non-empirical numerical quantity which quantifies the molecular structure and its branching pattern in many ways. In this point of view, the topological index can be regarded as a score function which maps each molecular structure to a real number and is used as a descriptor of the molecule under testing [13-16]. Topological indices give good predictions of the variety of physico-chemical properties of chemical compounds containing boiling point, the heat of evaporation, heat of formation, chromatographic retention times, surface tension, vapor pressure etc. Since the 1970s, two degree based graph invariants have been extensively studied. These are the first Zagreb index M1 and the second Zagreb index M2, introduced by Gutman and Trinajstic [17] and are defined as: $M_{1}(G)=\sum_{v \in V(G)}\left(\mathrm{d}_{v}\right)^{2}$ and $M_{2}(G)=\sum_{u v \in E(G)} \mathrm{d}_{u} \mathrm{~d}_{v}$.

Results obtained in the theory of Zagreb indices are summarized in the review [18].

Second modified Zagreb index is defined as: 


$$
{ }^{m} M_{2}(G)=\sum_{u v \in E(G)} \frac{1}{d_{u} d_{v}}
$$

In 1998, working independently, Bollobas and Erdos [19] and Amic et al. [20] proposed general Randic index. It has been extensively studied by both mathematicians and theoretical chemists (See, for example, [21,22]). The Randic index is defined as:

$$
R_{\alpha}(G)=\sum_{u v \in E(G)}\left(d_{u} d_{v}\right)^{\alpha}
$$

where $\alpha$ is an arbitrary real number.

Symmetric division index is defined as:

$$
S D D(G)=\sum_{u v \in E(G)}\left\{\frac{\min \left(d_{u}, d_{v}\right)}{\max \left(d_{u}, d_{v}\right)}+\frac{\max \left(d_{u}, d_{v}\right)}{\min \left(d_{u}, d_{v}\right)}\right\}
$$

Another variant of Randic index is the harmonic index defined as:

$$
H(G)=\sum_{v u \in E(G)} \frac{2}{d_{u}+d_{v}}
$$

The Inverse sum index is defined as:

$$
I(G)=\sum_{v u \in E(G)} \frac{d_{u} d_{v}}{d_{u}+d_{v}}
$$

The augmented Zagreb index is defined as:

$$
A(G)=\sum_{v u \in E(G)}\left\{\frac{d_{u} d_{v}}{d_{u}+d_{v}-2}\right\}^{3}
$$

and it is useful for computing heat of formation of alkanes [23,24]

These topological indices can be recovered from M-polynomial [4], see following table 1.

Table 1. Derivation of some degree-based topological indices from M-polynomial

\begin{tabular}{|l|c|}
\hline Topological Index & Derivation from $M(G ; x, y)$ \\
\hline First Zagreb & $\left.\left(D_{x}+D_{y}\right)(M(G ; x, y))\right|_{x=y=1}$ \\
\hline Second Zagreb & $\left.\left(D_{x} D_{y}\right)(M(G ; x, y))\right|_{x=y=1}$ \\
\hline Second Modified Zagreb & $\left.\left(S_{x} S_{y}\right)(M(G ; x, y))\right|_{x=y=1}$ \\
\hline General Randić $\alpha \in \mathbb{N}$ & $\left.\left(D_{x}^{\alpha} D_{y}^{\alpha}\right)(M(G ; x, y))\right|_{x=y=1}$ \\
\hline Symmetric Division Index & $\left.\left(D_{x} S_{y}+S_{x} D_{y}\right)(M(G ; x, y))\right|_{x=y=1}$ \\
\hline Harmonic Index & $2 S_{x} J(M(G ; x, y))_{x=1}$ \\
\hline
\end{tabular}




\begin{tabular}{|l|c|}
\hline Inverse sum Index & $\mathrm{S}_{x} \mathrm{~J} \mathrm{D}_{x} \mathrm{D}_{y}(\mathrm{M}(\mathrm{G} ; \mathrm{x}, \mathrm{y}))_{x=1}$ \\
\hline Augmented Zagreb Index & $\mathrm{S}_{x}{ }^{3} \mathrm{Q}_{-2} \mathrm{~J} \mathrm{D}_{x}{ }^{3} \mathrm{D}_{y}{ }^{3}(\mathrm{M}(\mathrm{G} ; \mathrm{x}, \mathrm{y}))_{x=1}$ \\
\hline
\end{tabular}

where $D_{x}=x \frac{\partial(f(x, y)}{\partial x}, D_{y}=y \frac{\partial(f(x, y)}{\partial y} \mathcal{S}_{x}=\int_{0}^{x} \frac{f(t, y)}{t} d t S_{y}=\int_{0}^{y} \frac{f(x, t)}{t} d t ? \mathcal{V}(f(x y))=f(x, x)$, ;

$$
Q_{\alpha}(f(x, y))=x^{\alpha} f(x, y) .
$$

In 2013, Shirdel et al. in [25] proposed "hyper-Zagreb index" which is also degree based index. Definition 2.Let $\mathrm{G}$ be a simple connected graph. Then the hyper-Zagreb index of $\mathrm{G}$ is defined as

$$
H M(G)=\sum_{u v \in E(G)}\left[\mathrm{d}_{u}+\mathrm{d}_{v}\right]^{2}
$$

In 2012 Ghorbani and Azimi [26] proposed two new variants of Zagreb indices.

Definition 3. Let $\mathrm{G}$ be a simple connected graph. Then the first multiple Zagreb index of $\mathrm{G}$ is defined as

$$
P M_{1}(G)=\prod_{u v \in E(G)}\left[\mathrm{d}_{u}+\mathrm{d}_{v}\right] .
$$

We refer [27] to the readers for more detail.

Definition 4.Let $\mathrm{G}$ be a simple connected graph. Then the second multiple Zagreb index of $\mathrm{G}$ is defined as

$$
P M_{2}(G)=\prod_{u v \in E(G)}\left[\mathrm{d}_{u}+\mathrm{d}_{v}\right]
$$

Definition 5. Let $\mathrm{G}$ be a simple connected graph. Then the first Zagreb polynomial of $\mathrm{G}$ is defined as

$$
M_{1}(G, x)=\sum_{u v \in E(G)} x^{\left[\mathrm{d}_{u}+\mathrm{d}_{v}\right]} .
$$

Definition 6. Let $\mathrm{G}$ be a simple connected graph. Then second Zagreb polynomial of $\mathrm{G}$ is defined as

$$
M_{2}(G, x)=\sum_{u v \in E(G)} x^{\left[\mathrm{d}_{u}+\mathrm{d}_{v}\right]} .
$$

In this article, we compute M-polynomial, first Zagreb polynomial and second Zagreb polynomial of Octagonal network shown in figure 1. We also compute some degree-based topological indices. 
We denote octagonal network by $O_{\{n, m\}}$ for $n, m \geq 2$. The planer representation of $O_{\{n, m\}}$ is shown in figure 1 with $\mathrm{m}$ rows and $\mathrm{n}$ columns of octagonal. Let $\mathrm{V}$ is the vertex set and $\mathrm{E}$ is the edge set of $O_{\{n, m\}} \cdot$ Then $V=\left\{x_{i}^{j} ; 1 \leq i \leq 2 n-1, i\right.$ is odd and $\left.1 \leq j \leq 3 m+1\right\} \cup\left\{x_{i}^{3 j-2} ; 1 \leq i \leq 2 n, i\right.$ is even and $\left.1 \leq \mathrm{j} \leq \mathrm{m}+1\right\}$ $\cup\left\{x_{2 n}^{3 j-1}, x_{2 n}^{3 j} ; 1 \leq j \leq m\right\}$

and $E=\left\{x_{i}^{j} x_{i}^{j+1} ; 1 \leq i \leq 2 n-1, i\right.$ is odd and $\left.1 \leq j \leq 3 m\right\} \cup\left\{x_{i}^{3 j-2} x_{i+1}^{3 j-2} ; 1 \leq i \leq 2 n-1, i\right.$ is odd and $\left.1 \leq \mathrm{j} \leq \mathrm{m}+1\right\}$ $\cup\left\{x_{i}^{3 j-2} x_{i+1}^{3 j-1} ; 1 \leq i \leq 2 n-2, i\right.$ is even and $\left.1 \leq \mathrm{j} \leq \mathrm{m}\right\} \cup\left\{x_{i}^{3 j} x_{i-1}^{3 j+1} ; 3 \leq i \leq 2 n-1, i\right.$ is odd and $\left.1 \leq \mathrm{j} \leq \mathrm{m}\right\}$ $\cup\left\{x_{2 n}^{j} x_{2 n}^{j+1} ; 1 \leq j \leq 3 m\right\}$

The number of vertices in octagonal network is $(4 m+2) n+2 m$ and number of edges in an octagonal network is $(6 m+1) n+m$.

\section{MAIN RESULTS}

In this part we give our main computational results.

Theorem 1. Let $O_{\{n, m\}}$ be the octagonal network. Then the M-polynomial of $O_{\{n, m\}}$ is $M\left(D P Z_{n}, x, y\right)=(2 n+2 m+4) x^{2} y^{2}+(4 n+4 m-8) x^{2} y^{3}+(6 m n-5 n-5 m+4) x^{3} y^{3}$. 


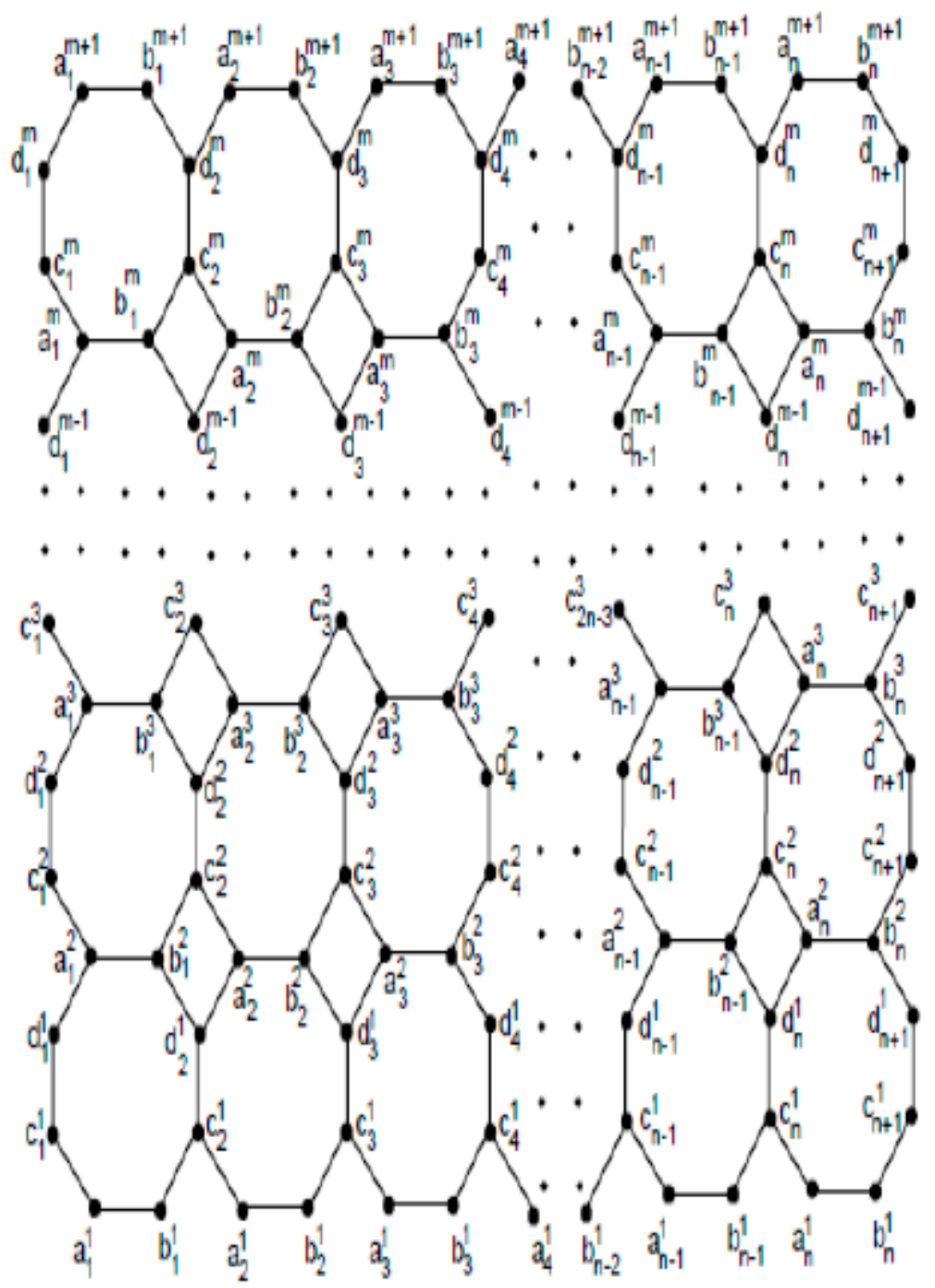

Figure 1. The Octagonal network $O_{\{n, m\}}$

Proof. Let $O_{\{n, m\}}$ be the octagonal network. The edge set $E\left(O_{\{n, m\}}\right)$ is divided into three edge partitions based on degrees of end vertices. The first edge partition $E_{1}\left(O_{\{n, m\}}\right)$ contains $2 n+2 m+4$ edges uv, where $d_{u}=d_{v}=2$. The second edge partition $E_{2}\left(O_{\{n, m\}}\right)$ contains $4 n+4 m-8$ edges uv, where $d_{u}=2, d_{v}=3$. The third edge partition $E_{3}\left(O_{\{n, m\}}\right)$ contains $6 m n-5 n-5 m+4$ edges uv, where $d_{u}=d_{v}=3$. From definition 1 the M-polynomial of $O_{\{n, m\}}$ is given by 


$$
\begin{aligned}
& M\left(O_{\{n, m\}} ? x, y\right)=\sum_{i \leq j} m_{i j} x^{i} y^{j} \\
& =\sum_{2 \leq 2} m_{22} x^{2} y^{2}+\sum_{2 \leq 3} m_{23} x^{2} y^{3}+\sum_{3 \leq 3} m_{33} x^{3} y^{3} \\
& =\sum_{u v \in} E_{1}\left(O_{\{n, m\}} m_{22} x^{2} y^{2}+\sum_{u v \in E_{2}\left(O_{\{n, m\}}\right)} m_{23} x^{2} y^{3}+\sum_{u v \in E_{3}\left(O_{\{n, m\}}\right)} m_{33} x^{3} y^{3}\right. \\
& =\left|E_{1}\left(O_{\{n, m\}}\right)\right| x^{2} y^{2}+\left|E_{2}\left(O_{\{n, m\}}\right)\right| x^{2} y^{3}+\left|E_{3}\left(O_{\{n, m\}}\right)\right| x^{3} y^{3} \\
& =(2 n+2 m+4) x^{2} y^{2}+(4 n+4 m-8) x^{2} y^{3}+(6 m n-5 n-5 m+4) x^{3} y^{3} .
\end{aligned}
$$
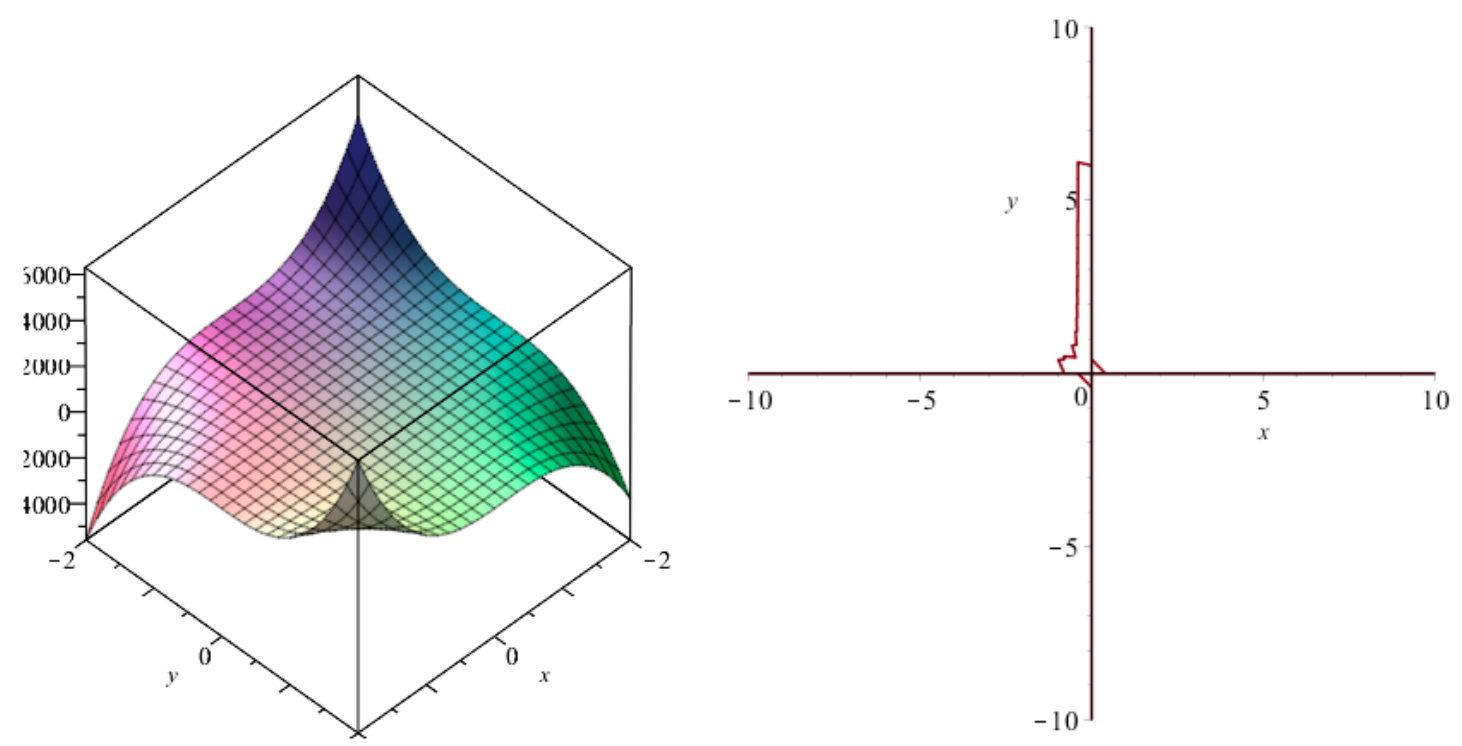

Figure 2. 3D and implicit plot of the M-polynomial of $O_{\{4,5\}}$

Now we compute some degree-based topological indices from this M-polynomial.

Proposition 2. Let $O_{\{n, m\}}$ be the octagonal network. Then

1. $M_{1}\left(O_{\{n, m\}}\right)=36 m n-2 m-2 n$.

2. $M_{2}\left(O_{\{n, m\}}\right)=54 m n-13 m-13 n+4$.

3. ${ }^{m} M_{2}\left(O_{\{n, m\}}\right)=\frac{11}{18} n+\frac{11}{18} m+\frac{1}{9}+\frac{2}{3} m n$.

4. $R_{\alpha}\left(O_{\{n, m\}}\right)=(2 n+2 m+4) 4^{a}+(4 n+4 m-8) 6^{a}+(6 m n-5 m-5 n+4) 9^{a}$.

5. $R_{\alpha}\left(O_{\{n, m\}}\right)=\frac{(2 n+2 m+4)}{4^{\alpha}}+\frac{(4 n+4 m-8)}{6^{\alpha}}+\frac{(6 m n-5 m-5 n+4)}{9^{\alpha}}$. 
6. $S S D\left(O_{\{n, m\}}\right)=\frac{8}{3} n+\frac{8}{3} m-\frac{4}{3}+12 m n$.

7. $H\left(O_{\{n, m\}}\right)=\frac{14}{15} n+\frac{14}{15} m+\frac{2}{15}+2 m n$.

8. $I\left(O_{\{n, m\}}\right)=-\frac{7}{10} n-\frac{7}{10} m+\frac{2}{5}+9 m n$.

9. $A\left(O_{\{n, m\}}\right)=-\frac{573}{64} n-\frac{573}{64} m+\frac{217}{16}+\frac{2187}{32} m n$

Proof. Let $M\left(O_{\{n, m\}} ; x, y\right)=f(x, y)=(2 n+2 m+4) x^{2} y^{2}+(4 n+4 m-8) x^{2} y^{3}+(6 m n-5 n-5 m+4) x^{3} y^{3}$.

Then

$$
\begin{aligned}
& D_{x} f(x, y)=2(2 n+2 m+4) x^{2} y^{2}+2(4 n+4 m-8) x^{2} y^{3}+3(6 m n-5 n-5 m+4) x^{3} y^{3}, \\
& D_{y} f(x, y)=2(2 n+2 m+4) x^{2} y^{2}+3(4 n+4 m-8) x^{2} y^{3}+3(6 m n-5 n-5 m+4) x^{3} y^{3}, \\
& D_{y} D_{x} f(x, y)=4(2 n+2 m+4) x^{2} y^{2}+6(4 n+4 m-8) x^{2} y^{3}+9(6 m n-5 n-5 m+4) x^{3} y^{3}, \\
& S_{y}(f(x, y))=\frac{(2 n+2 m+4)}{2} x^{2} y^{2}+\frac{(4 n+4 m-8)}{3} x^{2} y^{3}+\frac{(6 m n-5 n-5 m+4)}{3} x^{3} y^{3}, \\
& S_{x} S_{y}(f(x, y))=\frac{(2 n+2 m+4)}{4} x^{2} y^{2}+\frac{(4 n+4 m-8)}{6} x^{2} y^{3}+\frac{(6 m n-5 n-5 m+4)}{9} x^{3} y^{3} \text {, } \\
& D_{y}^{\alpha}(f(x, y))=2^{\alpha}(2 n+2 m+4) x^{2} y^{2}+3^{\alpha}(4 n+4 m-8) x^{2} y^{3}+3^{\alpha}(6 m n-5 n-5 m+4) x^{3} y^{3} \\
& D_{x}^{\alpha} D_{y}^{\alpha}(f(x, y))=2^{2 \alpha}(2 n+2 m+4) x^{2} y^{2}+2^{\alpha} 3^{\alpha}(4 n+4 m-8) x^{2} y^{3}+3^{2 \alpha}(6 m n-5 n-5 m+4) x^{3} y^{3} \\
& S_{y}^{\alpha}(f(x, y))=\frac{(2 n+2 m+4)}{2^{\alpha}} x^{2} y^{2}+\frac{(4 n+4 m-8)}{3^{\alpha}} x^{2} y^{3}+\frac{(6 m n-5 n-5 m+4)}{3^{\alpha}} x^{3} y^{3}, \\
& S_{x}{ }^{\alpha} S_{y}{ }^{\alpha}(f(x, y))=\frac{(2 n+2 m+4)}{2^{2 \alpha}} x^{2} y^{2}+\frac{(4 n+4 m-8)}{2^{\alpha} 3^{\alpha}} x^{2} y^{3}+\frac{(6 m n-5 n-5 m+4)}{3^{2 \alpha}} x^{3} y^{3}, \\
& S_{y} D_{x}(f(x, y))=(2 n+2 m+4) x^{2} y^{2}+\frac{2(4 n+4 m-8)}{3} x^{2} y^{3}+(6 m n-5 n-5 m+4) x^{3} y^{3}, \\
& S_{x} D_{y}(f(x, y))=(2 n+2 m+4) x^{2} y^{2}+\frac{3(4 n+4 m-8)}{2} x^{2} y^{3}+(6 m n-5 n-5 m+4) x^{3} y^{3}, . \\
& J f(x, y)=(2 n+2 m+4) x^{4}+(4 n+4 m-8) x^{5}+(6 m n-5 n-5 m+4) x^{6}, \\
& S_{x} J f(x, y)=\frac{(2 n+2 m+4)}{4} x^{4}+\frac{(4 n+4 m-8)}{5} x^{5}+\frac{(6 m n-5 n-5 m+4)}{6} x^{6}, \\
& J D_{x} D_{y} f(x, y)=4(2 n+2 m+4) x^{4}+6(4 n+4 m-8) x^{5}+9(6 m n-5 n-5 m+4) x^{6}, \\
& S_{x} J D_{x} D_{y} f(x, y)=(2 n+2 m+4) x^{4}+\frac{6(4 n+4 m-8)}{5} x^{5}+\frac{9(6 m n-5 n-5 m+4)}{6} x^{6},
\end{aligned}
$$


$D_{y}^{3} f(x, y)=2^{3}(2 n+2 m+4) x^{2} y^{2}+3^{3}(4 n+4 m-8) x^{2} y^{3}+3^{3}(6 m n-5 n-5 m+4) x^{3} y^{3}$,

$D_{x}^{3} D_{y}^{3} f(x, y)=2^{6}(2 n+2 m+4) x^{2} y^{2}+2^{3} 3^{3}(4 n+4 m-8) x^{2} y^{3}+3^{6}(6 m n-5 n-5 m+4) x^{3} y^{3}$,

$J D_{x}^{3} D_{y}^{3} f(x, y)=2^{6}(2 n+2 m+4) x^{4}+2^{3} 3^{3}(4 n+4 m-8) x^{5}+3^{6}(6 m n-5 n-5 m+4) x^{6}$,

$Q_{-2} J D_{x}^{3} D_{y}^{3} f(x, y)=2^{6}(2 n+2 m+4) x^{2}+2^{3} 3^{3}(4 n+4 m-8) x^{3}+3^{6}(6 m n-5 n-5 m+4) x^{4}$,

$S_{x}{ }^{3} J D_{x}{ }^{3} D_{y}{ }^{3} f(x, y)=2^{3}(2 n+2 m+4) x^{2}+2^{3}(4 n+4 m-8) x^{3}+\frac{3^{6}(6 m n-5 n-5 m+4)}{4^{3}} x^{4}$,

1. $M_{1}\left(O_{\{n, m\}}\right)=\left.\left(D_{x}+D_{y}\right) f(x, y)\right|_{x=y=1}=36 m n-2 m-2 n$.
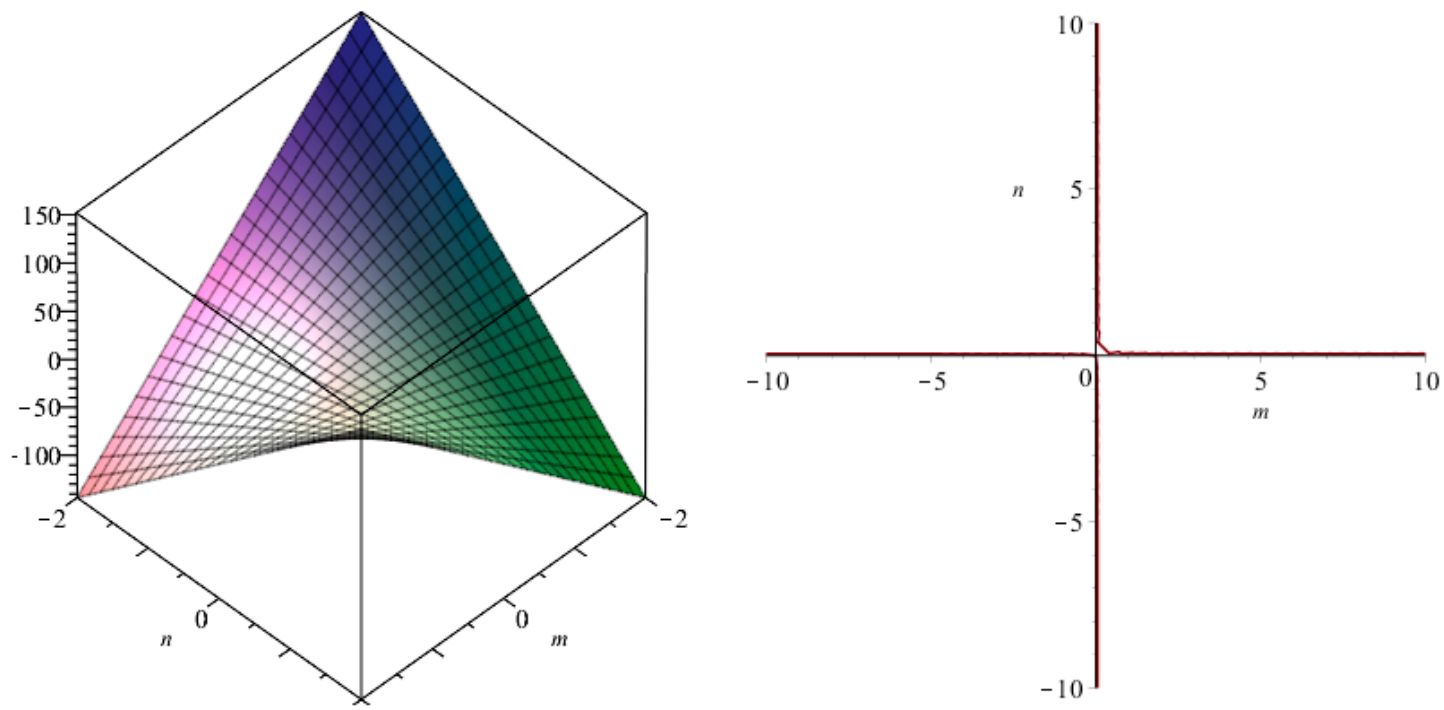

Figure 2. 3D and implicit plot for the first Zagreb index

2. $M_{2}\left(O_{\{n, m\}}\right)=\left.D_{y} D_{x}(f(x, y))\right|_{x=y=1}=54 m n-13 m-13 n+4$.
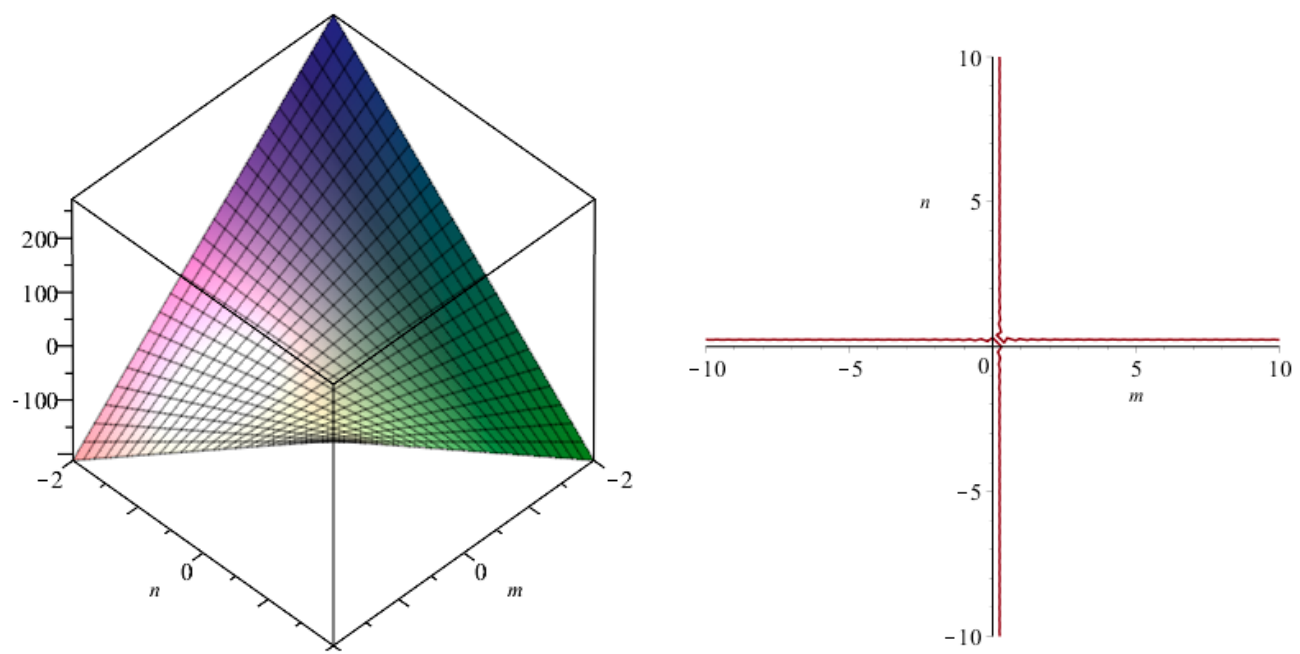

Figure 3. 3D and implicit plot for the second Zagreb index 
3.

$$
{ }^{m} M_{2}\left(O_{\{n, m\}}\right)=\left.S_{x} S_{y}(f(x, y))\right|_{x=y=1}=\frac{11}{18} n+\frac{11}{18} m+\frac{1}{9}+\frac{2}{3} m n .
$$
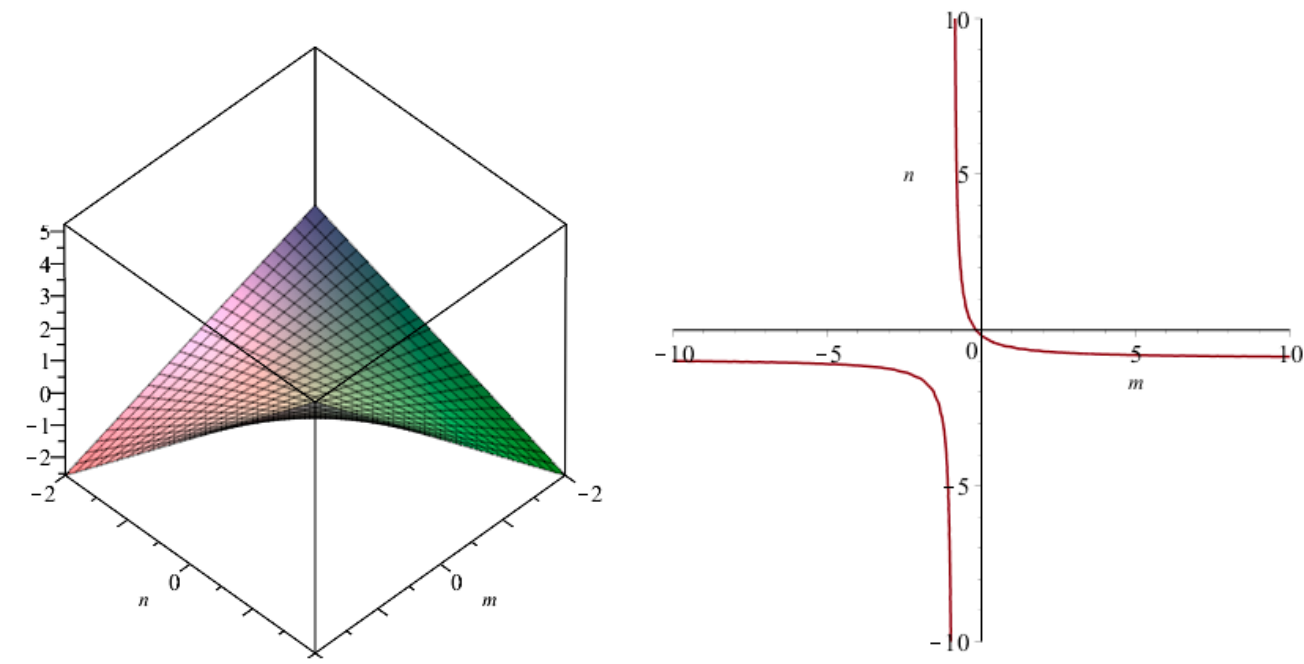

Figure 4. 3D and implicit plot for the modified second Zagreb index

4.

$R_{\alpha}\left(O_{\{n, m\}}\right)=\left.D_{x}^{\alpha} D_{y}^{\alpha}(f(x, y))\right|_{x=y=1}=(2 n+2 m+4) 4^{a}+(4 n+4 m-8) 6^{a}+(6 m n-5 m-5 n+4) 9^{a}$.
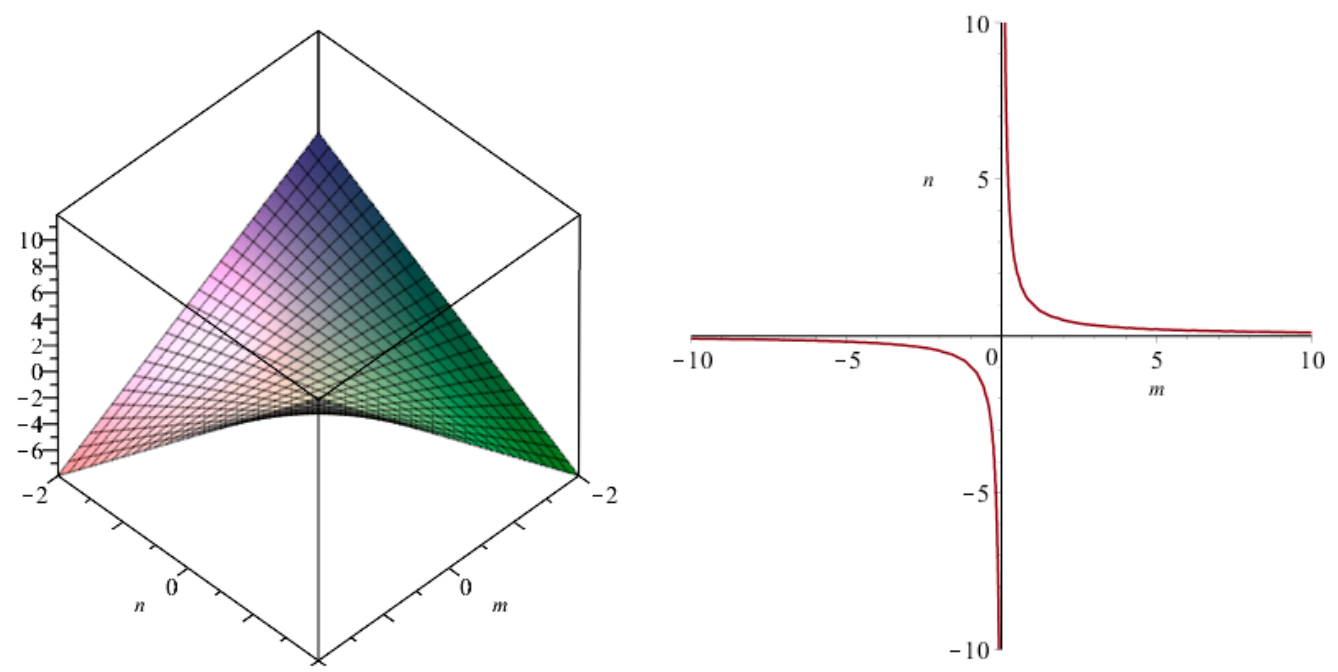

Figure 5. 3D and implicit plot for the Randic index for alpha=-1/2

5.

$$
R_{\alpha}\left(O_{\{n, m\}}\right)=\left.S_{x}^{\alpha} S_{y}^{\alpha}(f(x, y))\right|_{x=y=1}=\frac{(2 n+2 m+4)}{4^{\alpha}}+\frac{(4 n+4 m-8)}{6^{\alpha}}+\frac{(6 m n-5 m-5 n+4)}{9^{\alpha}} .
$$



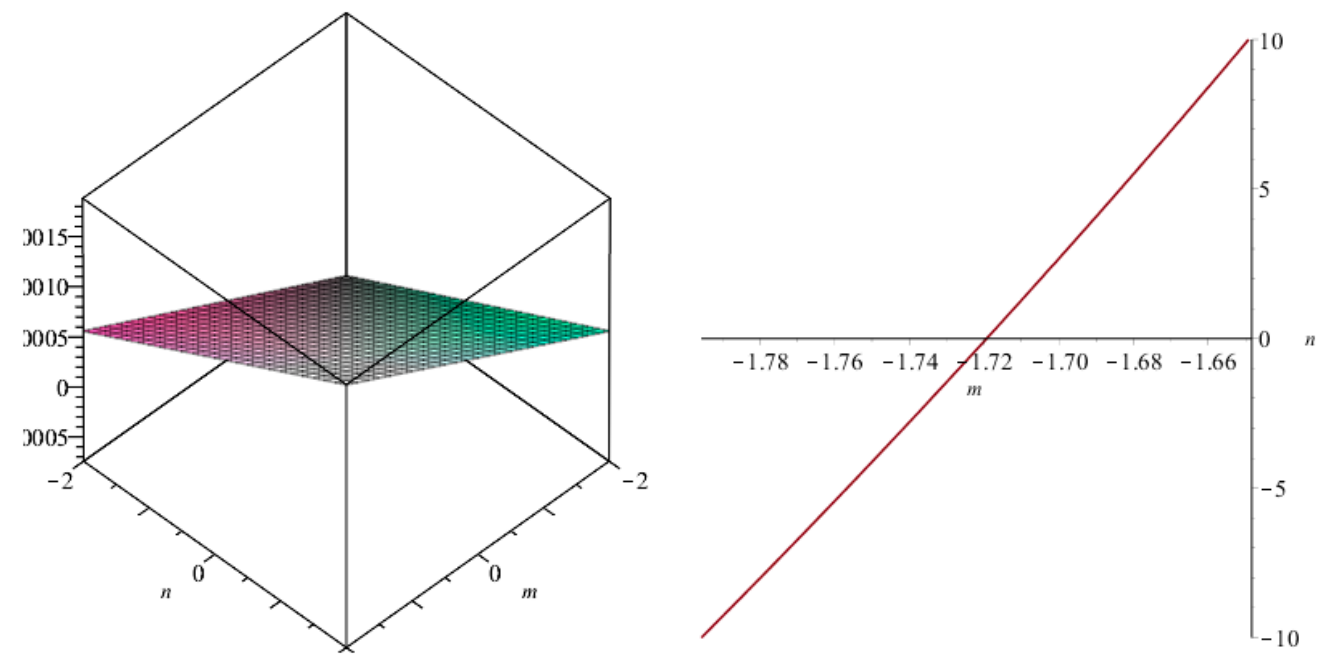

Figure 6. $3 \mathrm{D}$ and implicit plot for the generalized Randic index for alpha $=8$

6.

$$
\operatorname{SSD}\left(O_{\{n, m\}}\right)=\left.\left(S_{y} D_{x}+S_{x} D_{y}\right)(f(x, y))\right|_{x=y=1}=\frac{8}{3} n+\frac{8}{3} m-\frac{4}{3}+12 m n .
$$
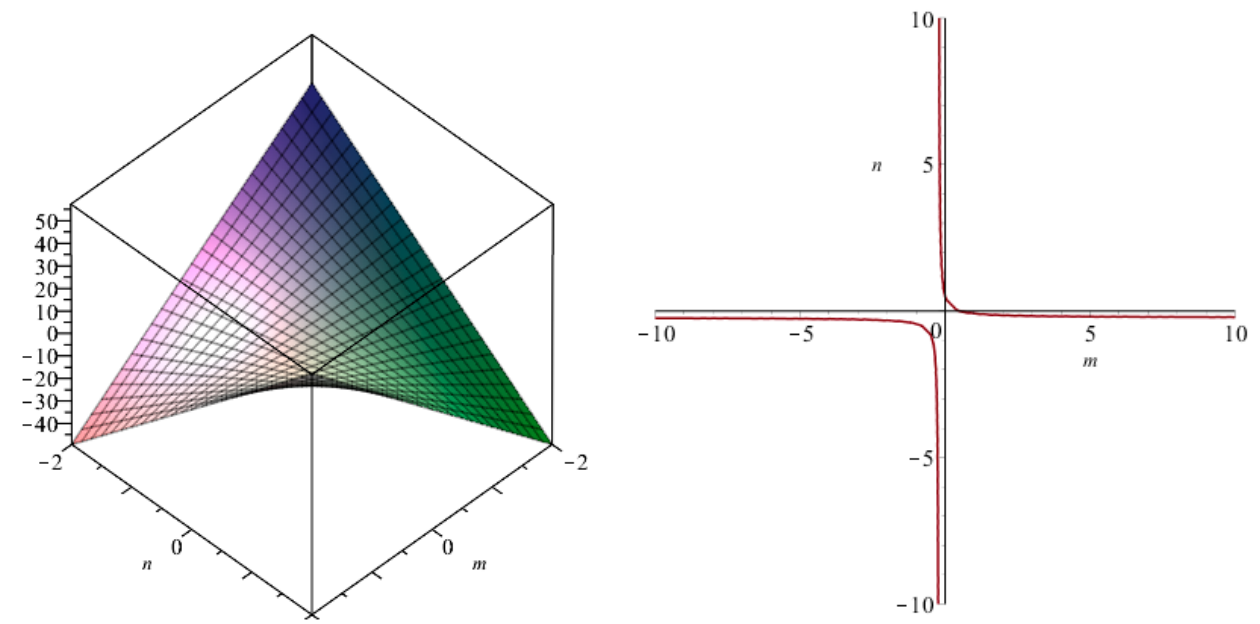

Figure 6. 3D and implicit plot for the Symmetric division index

7.

$H\left(O_{\{n, m\}}\right)=\left.2 S_{x} J(f(x, y))\right|_{x=1}=\frac{14}{15} n+\frac{14}{15} m+\frac{2}{15}+2 m n$. 

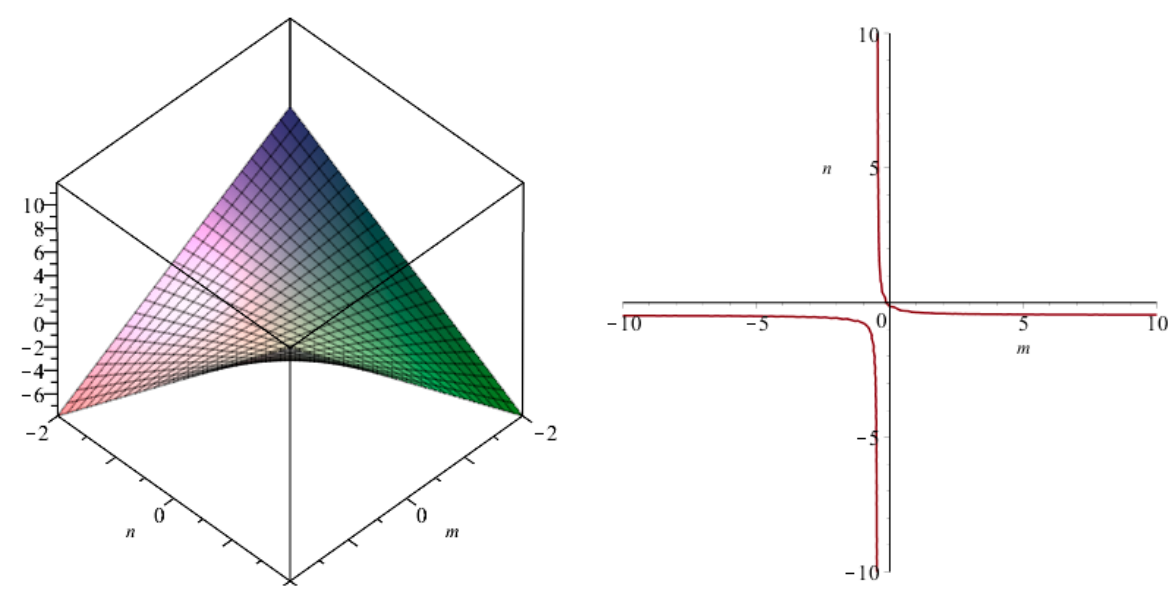

Figure 7. 3D and implicit plot for the Harmonic index

8.

$I\left(O_{\{n, m\}}\right)=S_{x} J D_{x} D_{y}(f(x, y))_{x=1}=-\frac{7}{10} n-\frac{7}{10} m+\frac{2}{5}+9 m n$.
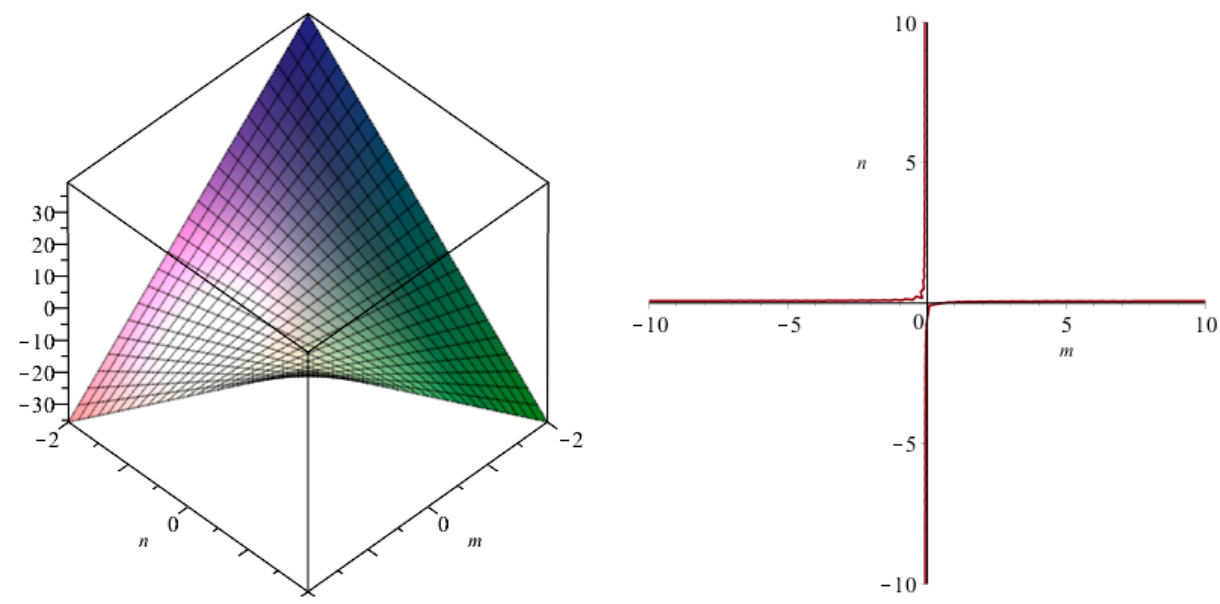

Figure 8. 3D and implicit plot for the Inverse Sum Index

9.

$$
A\left(O_{\{n, m\}}\right)=S_{x}^{3} Q_{-2} J D_{x}^{3} D_{y}^{3}(f(x, y))=-\frac{573}{64} n-\frac{573}{64} m+\frac{217}{16}+\frac{2187}{32} m n .
$$



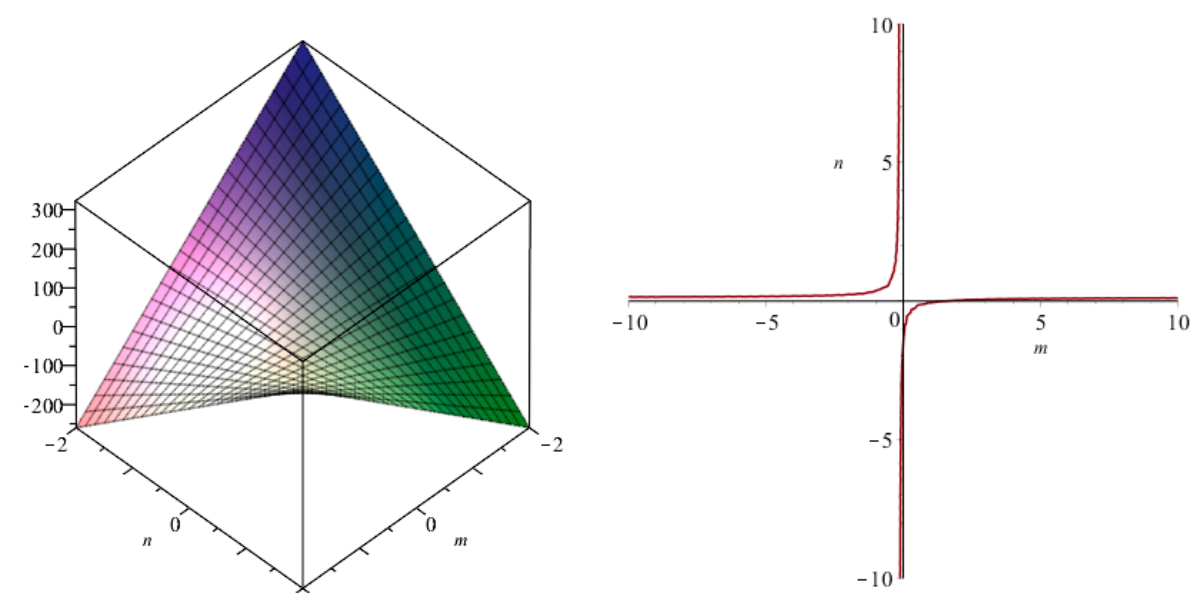

Figure 9. 3D and implicit plot for the Augmented Zagreb index

Theorem 3. Let $O_{\{n, m\}}$ be the octagonal network. Then the first and second Zagreb polynomials of $O_{\{n, m\}}$ are

1. 䏼 $\left(\mathfrak{O}_{\{n, m\}} x\right)=6 m n x^{6}-5 m x^{6}-5 n x^{6}+4 m x^{5}+4 n x^{5}+4 x^{6}+2 m x^{4}+2 n x^{4}-8 x^{5}+4 x^{4}$.

2. 䟠 $2\left(O_{\{n, m\}}, x\right)=6 m n x^{9}-5 m x^{9}-5 n x^{9}+4 x^{9}+4 m x^{6}+4 n x^{6}-8 x^{6}+2 m x^{4}+2 n x^{4}+4 x^{4}$.

Proof. Let $O_{\{n, m\}}$ be the octagonal network. Then

1. by the definition the first Zagreb polynomial (Definition 5)

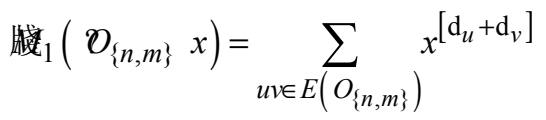

$$
\begin{aligned}
& =\sum_{u v \in E_{1}\left(O_{\{n, m\}}\right)} x^{\left[\mathrm{d}_{u}+\mathrm{d}_{v}\right]}+\sum_{u v \in E_{2}\left(O_{\{n, m\}}\right)} x^{\left[\mathrm{d}_{u}+\mathrm{d}_{v}\right]}+\sum_{u v \in E_{3}\left(O_{\{n, m\}}\right)} x^{\left[\mathrm{d}_{u}+\mathrm{d}_{v}\right]} \\
& \text { 爘 } E_{1}\left(O_{\{n, m\}}\right)\left|x^{4}+\right| E_{2}\left(O_{\{n, m\}}\right)\left|x^{5}+\right| E_{3}\left(O_{\{n, m\}}\right) \mid x^{6} \\
& =6 m n x^{6}-5 m x^{6}-5 n x^{6}+4 m x^{5}+4 n x^{5}+4 x^{6}+2 m x^{4}+2 n x^{4}-8 x^{5}+4 x^{4} \text {. }
\end{aligned}
$$



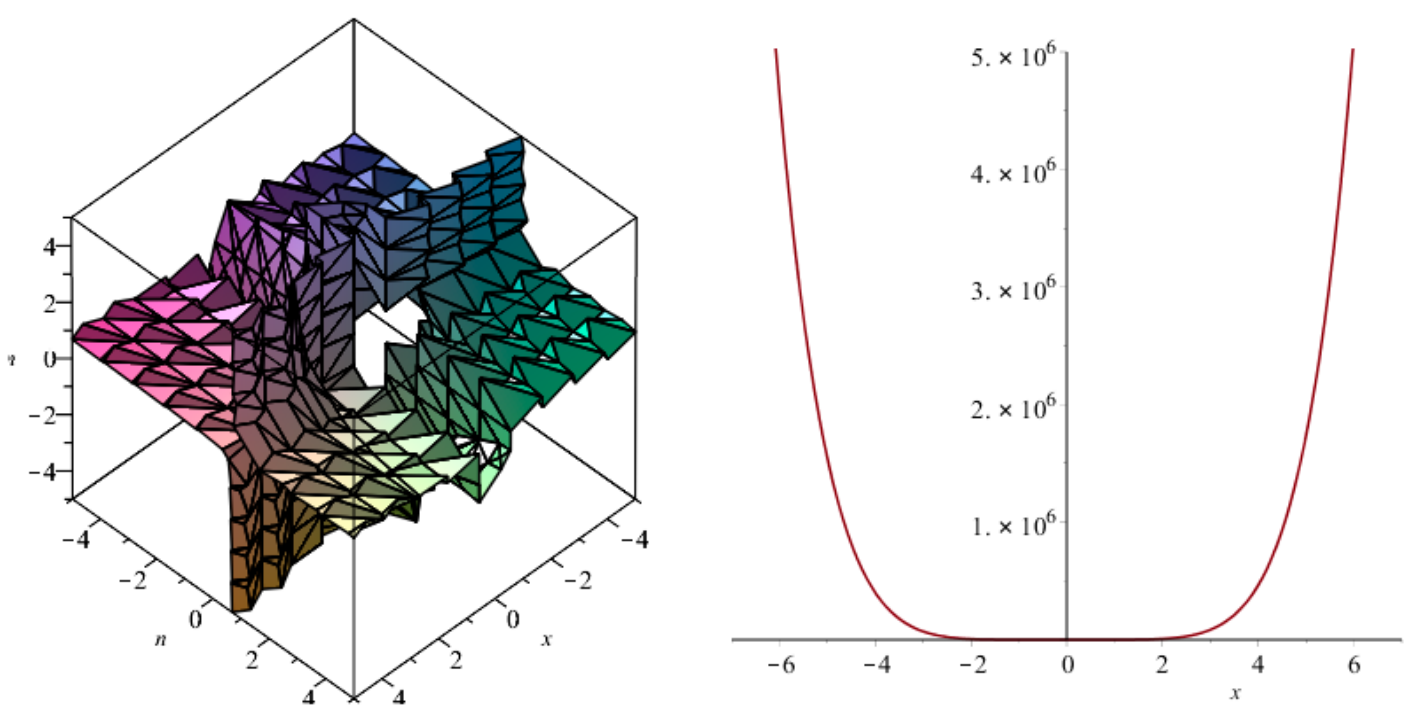

Figure 10. 3D implicit plot of first Zagreb polynomial and Interactive plot with two parameters 2. Now by definition the second Zagreb polynomial (Definition 6)

$$
\begin{aligned}
& \text { 脑 }_{2}\left(\mathfrak{D}_{\{n, m\}} x\right)=\sum_{u v \in E\left(O_{\{n, m\}}\right)} x^{\left[\mathrm{d}_{u} \times \mathrm{d}_{v}\right]} \\
&=\sum_{u v \in E_{1}\left(O_{\{n, m\}}\right)} x^{\left[\mathrm{d}_{u} \times \mathrm{d}_{v}\right]}+\sum_{u v \in E_{2}\left(O_{\{n, m\}}\right)} x^{\left[\mathrm{d}_{u} \times \mathrm{d}_{v}\right]}+\sum_{u v \in E_{3}\left(O_{\{n, m\}}\right)} x^{\left[\mathrm{d}_{u} \times \mathrm{d}_{v}\right]} \\
& \text { 爘 } \\
& E_{1}\left(O_{\{n, m\}}\right)\left|x^{4}+\right| E_{2}\left(\mathscr{D}_{\{n, m\}}\right)\left|x^{6}+\right| E_{3}\left(O_{\{n, m\}}\right) \mid x^{9} \\
&= 6 m n x^{9}-5 m x^{9}-5 n x^{9}+4 x^{9}+4 m x^{6}+4 n x^{6}-8 x^{6}+2 m x^{4}+2 n x^{4}+4 x^{4} .
\end{aligned}
$$
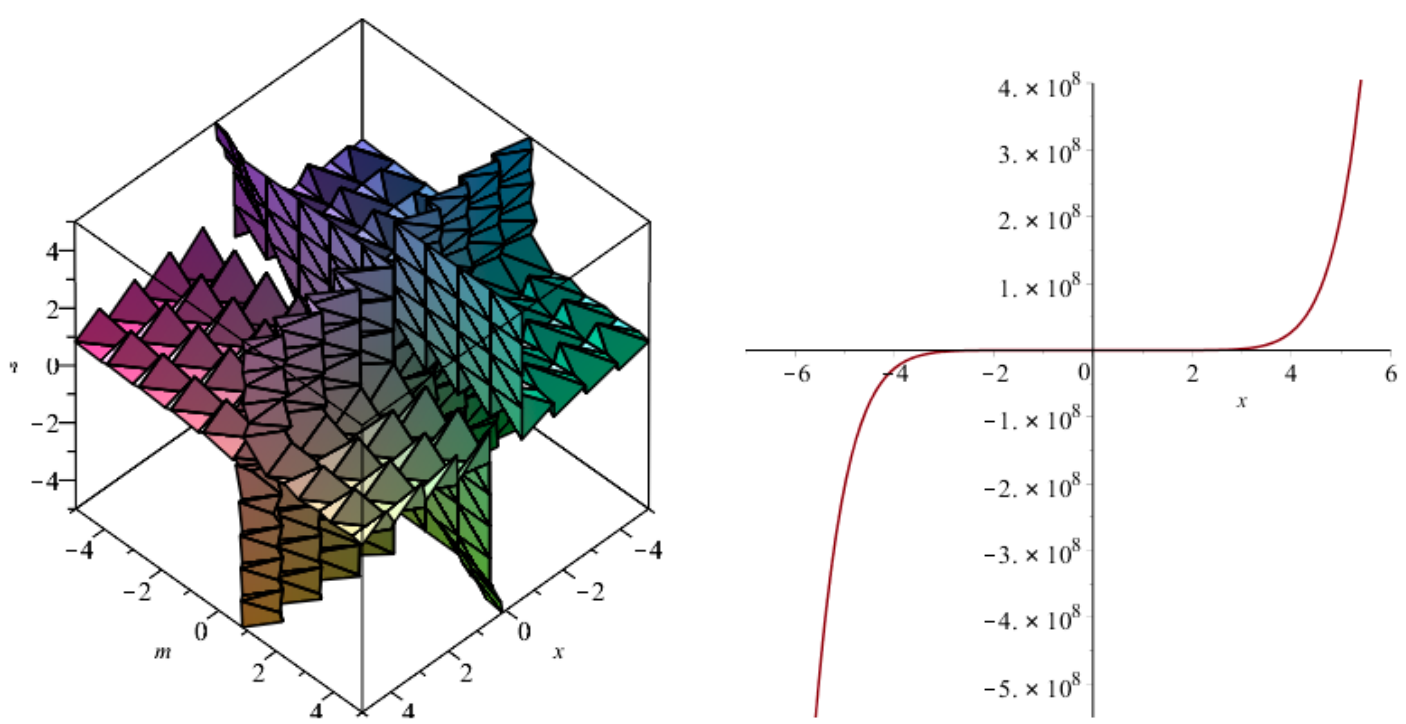

Figure 11. 3D implicit plot of second Zagreb polynomial and Interactive plot with two parameters Proposition 12. Let $O_{\{n, m\}}$ be the octagonal network. Then the hyper-Zagreb index, first multiple Zagreb index and the second multiple Zagreb index of $O_{\{n, m\}}$ are 
1. $H M\left(\mathcal{O}_{\{n, m\}}\right) \quad 216 m n-48 m-48 n+8$.

2. $P M_{1}\left(\mathcal{O}_{\{n, m\}}\right)=\frac{331776}{390625} \times 6656^{m n} \times 6^{-5 n-5 m} \times 10^{4 n+4 m}$.

3. $P M_{2}\left(\mathcal{O}_{\{n, m\}}\right)=31441^{m n} \times 3^{-10 n-10 m} \times 12^{4 n+4 m}$.

\section{Proof.}

1. By definition of hyper-Zagreb index (Definition 2)

$$
\begin{aligned}
H M\left(O_{\{n, m\}}\right) & =\sum_{u v \in E\left(O_{\{n, m\}}\right)}\left[d_{u}+d_{v}\right]^{2} \\
& =\sum_{u v \in E_{1}\left(O_{\{n, m\}}\right. \text { 哓 }}\left[d_{u}+d_{v}\right]^{2}+\sum_{u v \in E_{2}\left(O_{\{n, m\}}\right)}\left[d_{u}+d_{v}\right]^{2}+\sum_{u v \in E_{3}\left(O_{\{n, m\}}\right)}\left[d_{u}+d_{v}\right]^{2} \\
& =16 \mid E_{1}\left(O_{\{n, m\}} \text { 爘+25|}\left|E_{2}\left(\mathcal{O}_{\{n, m\}}\right)\right|+6\left|E_{3}\left(O_{\{n, m\}}\right)\right|\right. \\
& =16(2 n+2 m+4)+25(4 n+4 m-8)+36(6 m n-5 n-5 m+4) \\
& =216 m n-48 m-48 n+8 .
\end{aligned}
$$
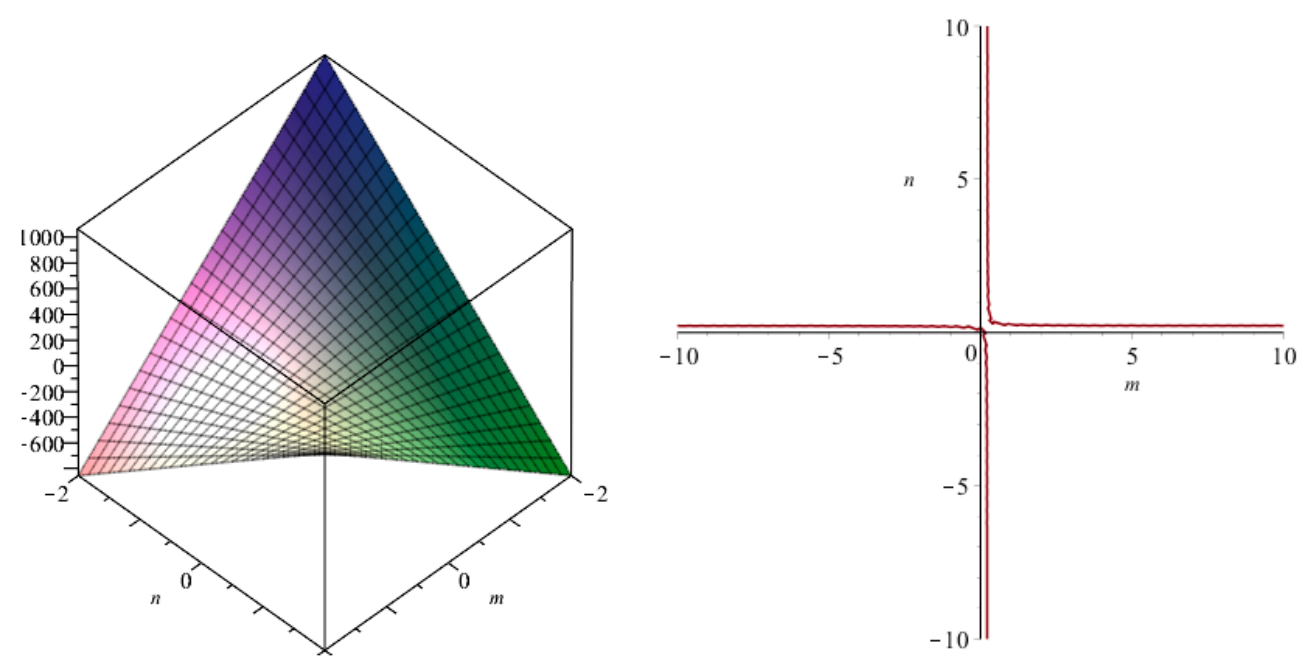

Figure 12. 3D and implicit plot for hyper-Zagreb index

2. By the definition of first multiple Zagreb index (Definition 3)

$$
\begin{aligned}
& \left.P M_{1}(\text { 蛪 } n, m\}\right)=\prod_{u v \in E(O\{n, m\})}\left[{ }_{u}+\mathrm{d}_{v}\right] \\
& =\prod_{\left.u v \in E_{1}(\text { 烧 } n, m\}\right)}\left[\mathrm{d}_{u}+\mathrm{d}_{v}\right] \times \prod_{u v \in E_{2}\left(O_{\{n, m\}}\right)}\left[\mathrm{d}_{u}+\mathrm{d}_{v}\right] \times \prod_{\left.u v \in E_{3}(\text { 螣 } n, m\}\right)}\left[\mathrm{d}_{u}+\mathrm{d}_{v}\right]
\end{aligned}
$$

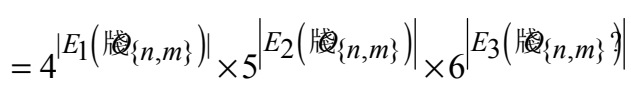

$$
\begin{aligned}
& =4^{2 n+2 m+4} \times 5^{4 n+4 m-8} \times 6^{6 m n-5 n-5 m+4} \\
& =\frac{331776}{390625} \times 46656^{m n} \times 6^{-5 n-5 m} \times 10^{4 n+4 m} \text {. }
\end{aligned}
$$



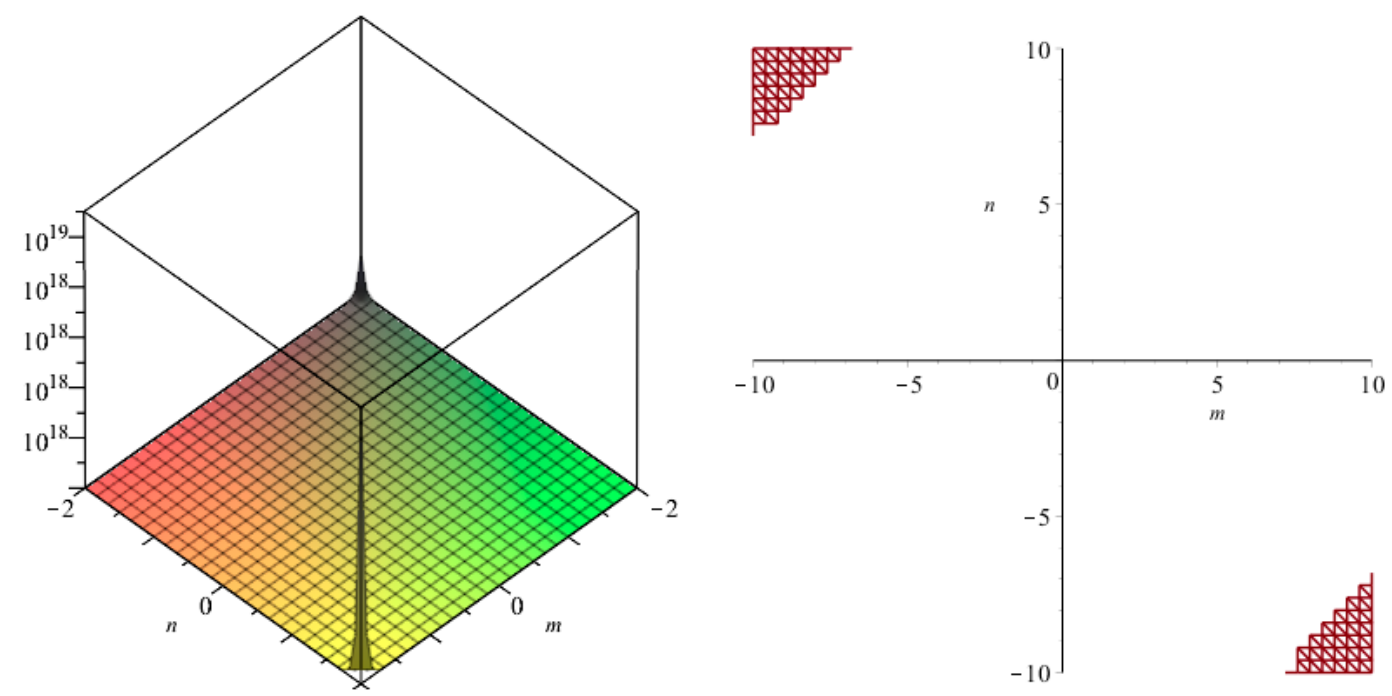

Figure 13. 3D and implicit plot for first multiple Zagreb index

3. By the definition of second multiple Zagreb index (Definition 4)

$$
\begin{aligned}
& P M_{2}\left(\text { 㸌 }_{n, m\}}\right)=\prod_{u v \in E\left(O_{\{n, m\}}\right)}\left[{ }_{u} \times \mathrm{d}_{v}\right]
\end{aligned}
$$

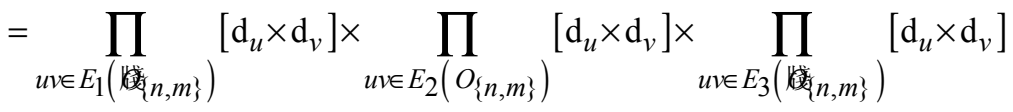

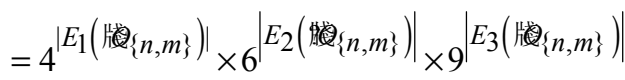

$$
\begin{aligned}
& =4^{2 n+2 m+4} \times 6^{4 n+4 m-8} \times 9^{6 m n-5 n-5 m+4} \\
& =531441^{m n} \times 3^{-10 n-10 m} \times 12^{4 n+4 m} \text {. }
\end{aligned}
$$
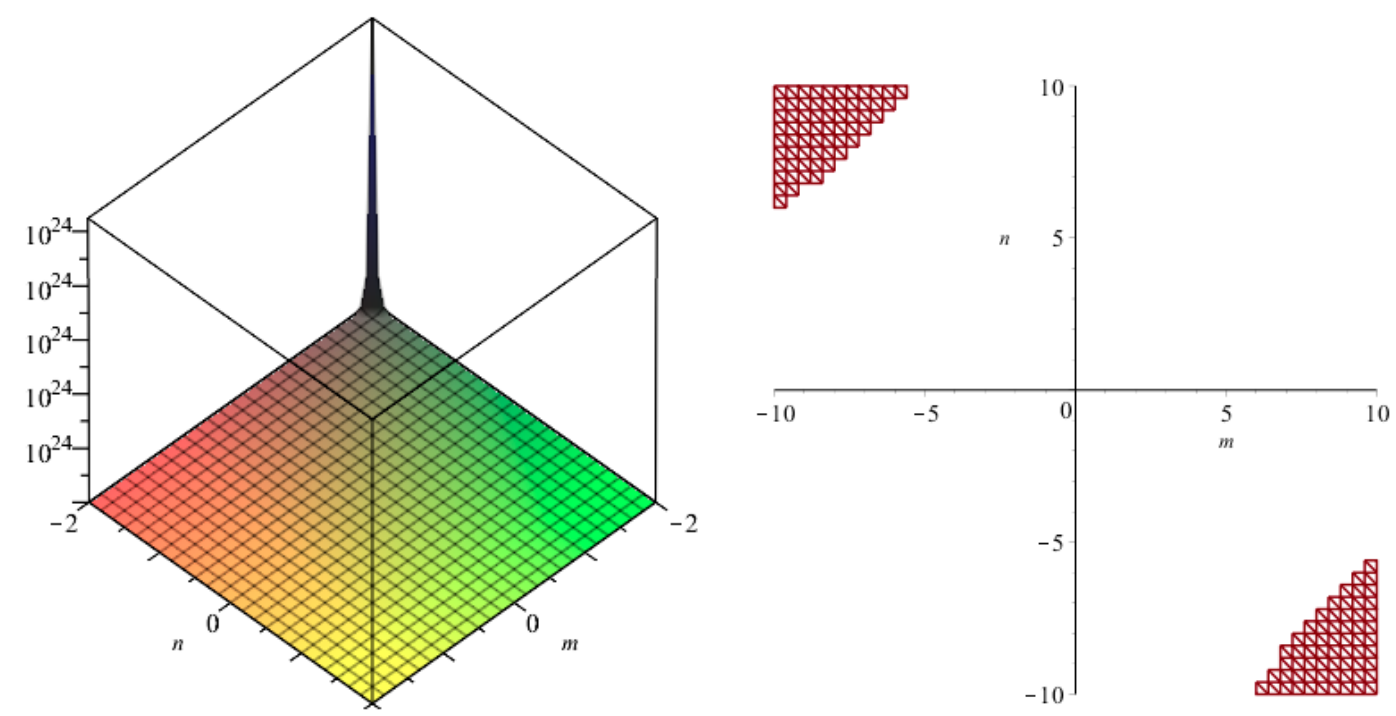

Figure 14. 3D and implicit plot for second multiple Zagreb index 


\section{CONCLUSIONS AND DISCUSSION}

In this article we computed many topological indices for the octagonal network. At first, we gave the general closed form of M-polynomial of this family and recover many degree-based topological indices out of it. We also computed multiple Zagreb indices and Zagreb polynomials of the octagonal network. We also gave graphs for the computed polynomials and topological indices see figure 2-14. Our graphical representations of topological indices show the dependency of the certain index on the structure. These results can play a vital role in determining properties of this network and its uses in industry, electronics, and pharmacy.

Acknowledgments: This research is supported by Gyeongsang National University, Jinju 52828, Korea

Author Contributions: Y. C. K. and W. N designed the problem and analysis tool; S. M. K. investigated and produced the initial results; W. N. and M. M. verified and improved the final results and wrote the paper.

Conflicts of Interest: “The authors declare no conflict of interest."

\section{References}

1. Stover, D.; Normile, D. Buckytubes, Popular Science; Apr 92, 240(4) p31,204063236

2. The many faces of nanotech, Nature Nanotechnology;Oct2007, Vol. 2 Issue 10, p585, 32096988

3. West, D. B. An Introduction to Graph Theory. (1996). Prentice-Hall.

4. Deutsch, E.; Klavzar, S. M-Polynomial and Degree-Based Topological Indices. Iranian Journal of Mathematical Chemistry, (2015) 6(2), 93-102.

5. Gutman, I. Molecular graphs with minimal and maximal Randic indices. Croatica Chem. Acta (2002)75, 357-369.

6. Gutman, I. Degree-based topological indices. Croat. Chem. Acta (2013)86, 351-361.

7. Vuki 'cevi'c, D. On the edge degrees of trees. Glas. Mat. Ser. III (2009)44(64) 259-266.

8. Gutman, I. Gutman, Some properties of the Wiener polynomials, Graph Theory Notes, New York 25 (1993) 13-18.

9. Munir, M.; Nazeer, W.; Rafique, S.; Nizami, A. R.; Kang, S. M. M-polynomial and degree-based topological indices of Titania Nanotubes, Symmetry 2016, 8, 117; doi:10.3390/sym8110117.

10. Munir,M.; Nazeer,W.; Rafique, S.; Kang, S. M. M-polynomial and degree-based topological indices of Buckytubes,(Submitted)

11. Kang, S.; Munir, M.; Nizami, A.; Shahzadi, Z.; Nazeer, W. Some Topological Invariants of the Möbius Ladder,Preprints 2016, 2016110040 (doi: 10.20944/preprints201611.0040.v1).

12. Munir, M.; Nazeer, W.; Rafique, S.; Nizami, A.; Kang, S. M. Some Computational Aspects of Triangular Boron Nanotubes, Preprints 2016, 2016110041 (doi: 10.20944/preprints201611.0041.v1).

13. Rucker, G.; Rucker, C. On topological indices, boiling points, and cycloalkanes. J. Chem. Inf. Comput. Sci. (1999) 39, 788.

14. Klavžar, S.; Gutman, I. A Comparison of the Schultz Molecular Topological Index with the Wiener Index. J. Chem. Inf. Comput.Sci. (1996) 36, 1001-1003

15. Deng H,Huang G, Jiang X A unified linear-programming modeling of some topological indices. J. Comb. Opt. (To appear) DOI 10.1007/s10878-013-9672-2.

16. Deng, H.; Yang, J. Xia F A general modeling of some vertex-degree based topological indices in benzenoid systems and phenylenes. Comp. Math. Appl. (2011) 61, 3017-3023.

17. Gutman, I.; Trinajstic, N. Graph theory and molecular orbitals total _-electron energy of alternant hydrocarbons. Chem. Phys. Lett. 1972, 17, 535-538. 
18. Gutman, I.; Das, K.C. The first Zagreb indices 30 years after. MATCH Commun. Math. Comput. Chem. 2004, 50, 83-92.

19. Bollobas, B.; Erdös, P. Graphs of extremal weights. Ars Combin. 1998, 50, 225-233.

20. Amic, D.; Beslo, D.; Lucic, B.; Nikolic, S.; Trinajsti'c, N. The Vertex-Connectivity Index Revisited. J. Chem. Inf Comput. Sci. 1998, 38, 819-822.

21. Kier, L.B.; Hall, L.H. Molecular Connectivity in Structure-Activity Analysis; John Wiley \& Sons: New York, NY, USA, 1986.

22. Li, X.; Gutman, I. Mathematical Aspects of Randic-Type Molecular Structure Descriptors; Mathematical Chemistry Monographs, No. 1; University of Kragujevac: Kragujevac, Serbia, 2006.

23. Yufei Huang, Bolian Liut, Lu Gan, Augmented Zagreb Index of Connected Graphs,MATCH Commun. Math. Comput. Chem. 67 (2012) 483-494

24. B. Furtula, A. Graovac, D. Vukicevi'c, Augmented Zagreb index, J. Math. Chem. 48 (2010) 370380.

25. Shirdel, G. H, Pour, H. R, Sayadi, A. M. The hyper-Zagreb index of graph operations, Iran J Math Chem 2013;4(2): 213-20

26. M. Ghorbani, N. Azimi, Note on multiple Zagreb indices, Iran. J. Math. Chem. 3 (2) (2012) 137-143.

27. Li, X.; Shi, Y. A Survey on the Randic Index. MATCH Commun. Math. Comput. Chem. 2008, 59, 127-156.

(C) 2016 by the authors; licensee Preprints, Basel, Switzerland. This article is an open access article distributed under the terms and conditions of the Creative Commons by Attribution (CC-BY) license (http://creativecommons.org/licenses/by/4.0/). 